\title{
Operations of the Pierre Auger Observatory
}

\section{Rossella Caruso ${ }^{a, *}$ on behalf of the Pierre Auger ${ }^{b}$ Collaboration}

(a complete list of authors can be found at the end of the proceedings)

${ }^{a}$ Dipartimento di Fisica e Astronomia "Ettore Majorana”, Università degli Studi di Catania \& INFN, Sezione di Catania, Catania, Italy

${ }^{b}$ Observatorio Pierre Auger, Av. San Martín Norte 304, 5613 Malargüe, Argentina

E-mail: spokespersons@auger.org

The construction of the first stage of the Pierre Auger Observatory, designed for research of ultra-high energy cosmic rays, began in 2001 with a prototype system. The Observatory has been collecting data since early 2004 and was completed in 2008. The Observatory is situated at $1400 \mathrm{~m}$ above sea level near Malargüe, (Mendoza province) in western Argentina, covering a vast plain of $3000 \mathrm{~km}^{2}$, known as the Pampa Amarilla. The Observatory consists of a hybrid detector, in which there are 1660 water-Cherenkov stations, forming the Surface Detector (SD) and 27 peripheral atmospheric fluorescence telescopes, comprising the Fluorescence Detector (FD). Over time, the Auger Observatory has been enhanced with different R\&D prototypes and is recently being to an important upgrade called AugerPrime. In the present contribution, the general operations of the SD and FD will be described. In particular the FD shift procedure - executable locally in Malargüe or remotely by teams in control rooms abroad within the Collaboration - and the newly SD shifts (operating since 2019) will be explained. Additionally, the SD and FD maintenance campaigns, as well as the data taking and data handling at a basic level, will be reported.

$37^{\text {th }}$ International Cosmic Ray Conference (ICRC 2021)

July 12th - 23rd, 2021

Online - Berlin, Germany

\footnotetext{
${ }^{*}$ Presenter
} 


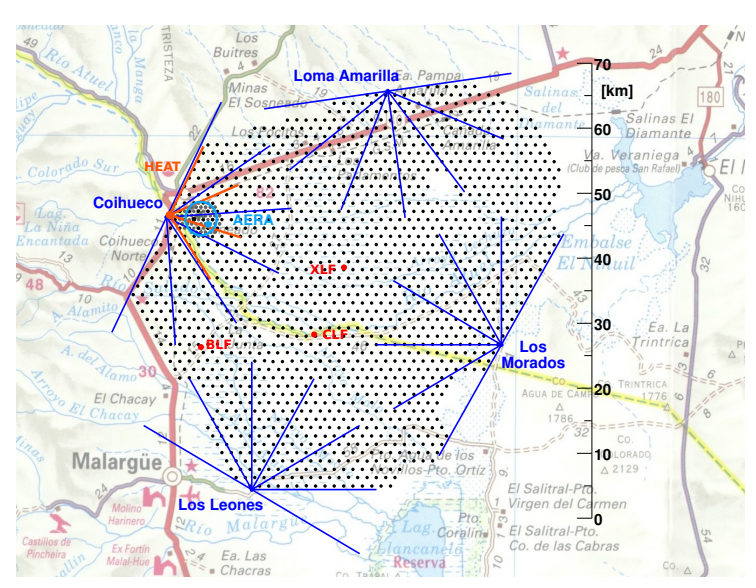

Figure 1: Map of the Pierre Auger Observatory. The dots represent the position of each detector in the SD Array; the Los Leones, Coihueco, Loma Amarilla and Los Morados labels represent the geographical denominations of the four sites, each grouping 6 FD telescopes, with blue lines indicating their field of view.

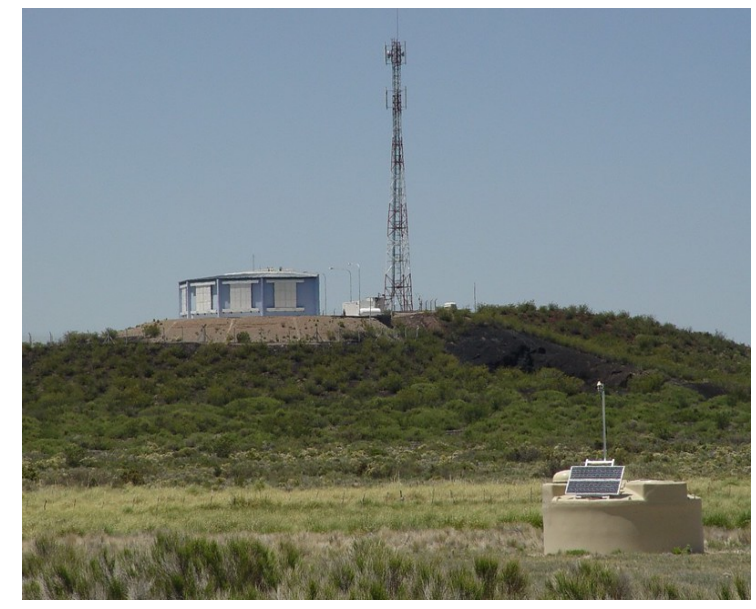

Figure 2: Photograph of the natural hill (Cerro Los Leones) where one of the four buildings, accommodating six atmospheric fluorescence telescopes, overlooks the surrounding area where water-Cherenkov detectors are placed, as the one shown in the bottom right of the figure.

\section{Overview of the Pierre Auger Observatory}

Ultra High Energy Cosmic Rays (UHECRs) are particles and nuclei from space with energies varying from $10^{18} \mathrm{eV}$ beyond $10^{20} \mathrm{eV}$. Striking the terrestrial atmosphere, they initiate Extensive Air Showers (EAS), secondary subatomic particles produced by their collision with atomic nuclei of air molecules [1]. The Pierre Auger Observatory was designed for measuring the UHECRs flux, arrival direction distribution and mass composition by detecting the EAS with a very high statistics and precision. Today, around 400 physicists from 93 institutions in 18 participating countries around the world are collaborating. Construction of the first stage of the Observatory began in 2001 with a prototype system [2]. The Observatory has been collecting data since early 2004 and it was completed in 2008. The Observatory [3] is located near Malargüe, in the Mendoza province of western Argentina, covering a vast plain of $3000 \mathrm{~km}^{2}$ known as the Pampa Amarilla, between $35.0^{\circ} \div 35.3^{\circ} \mathrm{S}$ latitude and $69.0^{\circ} \div 69.4^{\circ} \mathrm{W}$ longitude at an altitude of $1400 \mathrm{~m}$ (atmospheric depth of $\sim 875 \mathrm{~cm}^{2}$. This location was selected due to the low population density $\left(<0.1 \mathrm{~km}^{-2}\right)$, special climate (dry with clear skies), low pollution and flatness of landscape. The Observatory consists of the Fluorescence Detector (FD), 24 atmospheric fluorescence telescopes situated in four sites, on the top of natural hills (their geographical denominations: Cerro Los Leones, Coihueco, Los Morados, Loma Amarilla) overlooking the Surface Detector Array (SD) (Fig.2). Three additional atmospheric fluorescence telescopes with a higher viewing angle are located at Coihueco site, called HEAT (High Elevation Auger Telescopes). The SD consists in 1600 water-Cherenkov detectors (WCDs) deployed on a triangular grid of $1.5 \mathrm{~km}$ spacing (Fig.1). 61 additional WCDs infilled array with $750 \mathrm{~m}$ spacing together with HEAT extend the sensitivity down to $10^{17} \mathrm{eV}$. Over time the Observatory has been enhanced with different R\&D prototypes and recently subjected to a major upgrade (AugerPrime)[4]. 


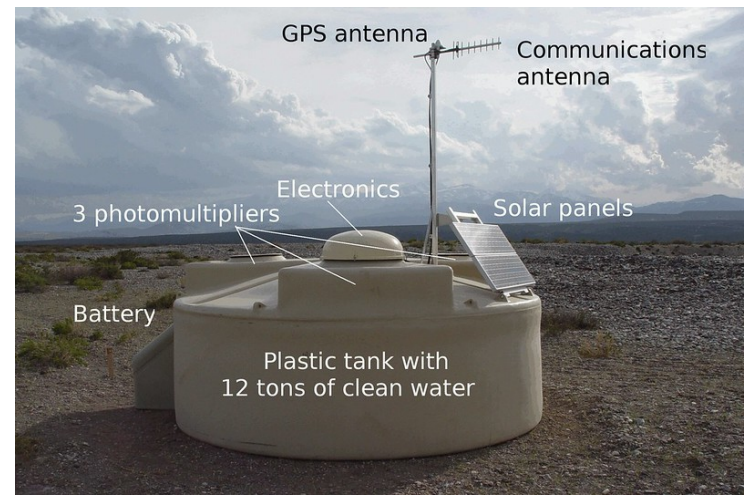

Figure 3: A water-Cherenkov detector with a schematic view of its components.

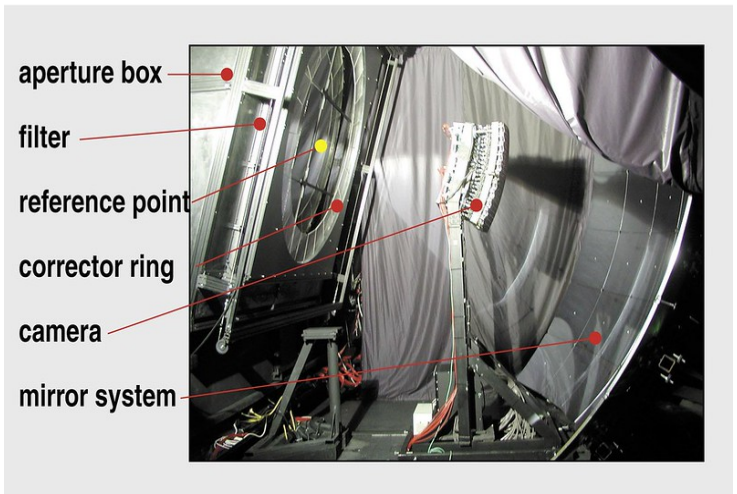

Figure 4: An atmospheric fluorescence telescope with a schematic view of its components.

Each water-Cherenkov detector [5](Fig.3) is a plastic tank of $10 \mathrm{~m}^{2}$ area and $1.5 \mathrm{~m}$ height containing 12000 liters of ultra purified water contained in a Tyvek reflective liner. The Cherenkov light generated by charged particles of the EAS crossing the tank is registered by three 9-in. diameter Photonis XP1805/D1 photomultiplier tubes (PMTs). A solar energy unit and batteries provide the required $10 \mathrm{~W}$ electrical power. A radio communication system, a GPS receiver with corresponding antenna, serves for event timing, data transmission and communication synchronization achieved via a custom wireless system. The SD front-end, read-out and data-acquisition electronics (SDE) supplies the PMTs, shapes and digitizes the output signal and generates threshold and triggers.

Each atmospheric fluorescence telescope [6] (Fig.4) records the fluorescence light isotropically emitted by the atmospheric $\mathrm{N}_{2}$ excited by the charged particles as well as the Cherenkov and scattered light produced during the EAS development. The telescope consists of a Schmidt optical system: a circular (1.1 m radius) diaphragm at the centre of curvature of a large spherical mirror of about $3.4 \mathrm{~m}$ radius of curvature. An ultra-violet transmitting filter, allowing the passage of the UV light, and a ring of annular corrector lenses, doubling the effective aperture and correcting the optical aberrations, are installed at the aperture. A camera of $4401.51^{\circ}$ hexagonal Photonis XP3062 PMTs, with an overall $\left(30^{\circ} \times 28.6^{\circ}\right)$ field of view, detects the fluorescence light focused by the mirror. Externally, a shutter system protects the telescope from the excessive outside light and atmospheric agents and a fail-safe curtain, mounted behind the diaphragm, protects the camera in case of malfunction of the shutters. The FD front-end, read-out and data-acquisition electronics (FDE) supplies the PMTs, shapes and digitizes the output signal, generates threshold and triggers. A computer (PC) is associated with each single telescope and connected via LAN to the site central readout computer $(E y e P C)$ that provides connection between communications network and single PCs. The events surviving all trigger levels are sent to the EyePC which generates a trigger for the SD. A Central Data Acquisition System (CDAS) combines the triggers from the SD Array with triggers from the FD sites and organizes the whole storage of data. The combined use of two different techniques - the so-called hybrid technique - allows unprecedented accuracy in reconstructing EAS from UHECRs. The bulk of data are provided by the SD having nearly $100 \%$ duty-cycle while the FD has only a $15 \%$ duty-cycle since it can only work at clear moonless nights. 


\section{Operations and maintenance of the detectors: the SD Array}

The SD consists of very robust detectors, designed to function autonomously. Each detector once built: tank placed in the field, filled with water and fitted with electronics at the deployment is a stand-alone unit, operating continuously with its own electronics and communication system. The SD data-taking runs non-stop and consequently the presence of a staff of shifters on site is not required. The operation of the whole SD is monitored online and different alarms are set on various parameters [7] so that if they detect an urgent problem the local staff intervenes for maintenance service. Recently, specific regular shifts have been set up for the monitoring. They are performed remotely within the Auger collaboration (Sec.5). Over more than 15 years of data-taking, the SD Array has been functioning stably with a duty cycle higher than 95\% [9]. However the detectors are exposed to very severe environmental conditions in the field: thermal variations, humidity, wind, salinity, dust, flooding, ground erosion (Fig.5); damages caused by local fauna and human vandalism or theft acts as well and, consequently, they undergo recurring maintenance. Overall, the SD maintenance is performed primarily by the Observatory permanent staff of engineers and technicians with the help of expert technicians and physicist from other Auger institutions, specifically for the PMTs and electronics maintenances. More generally, the detectors out of operation are named as Black Tanks (a detector expected to send trigger signal to CDAS but that has not sent any during the last 24 hours) and Grey Tanks (a detector where only one PMT out of the 3 is working). A very large number of Black Tanks is due to general problems of power or communication. To keep the number of malfunctioning detectors around 40-50, about 3 field trips per week are required and periodical maintenance trips, especially for PMTs (named PMT Weeks), are conducted. Nowadays the achieved number of stations out of order is about 4048. In particular, concerning the detectors themselves (tanks, liners, water), only very few failures have been detected over the operation time of the Observatory. The tanks are robust, uniform plastic containers, with additives to enhance ultraviolet protection, able to withstand effectively the environmental conditions. The liners are durable and flexible plastic cylindrical shells. Only a few liners leaked shortly after installation. In such worst case, the tank was emptied, removed from the field and replaced. The ultra-pure water, produced at a water plant at the Observatory at deployment (with a resistivity $\geq 15 \mathrm{M} \Omega \mathrm{cm}$ ), has shown no bacteria growth during the years as special tests indicated. The commercial batteries, charged by solar panels, have a lifetime affected by the temperature variations, thus for this reason they were installed on the shaded side of the tank and encapsulated in a robust protection. There is always enough reserve on the solar power system; just twice in the lifetime of the Observatory (for a period of 13 days: May $10^{\text {th }} \div 22^{\text {nd }}$, 2016 and, very recently, for a couple of days: June $19^{t h} \div 20^{t h}, 2021$ ) due to a long rainy and cloudy period, a total discharge of batteries occurred. Studies of the power system show a perfectly normal behavior: the average battery lifetime is 6 years [8], confirming initial expectations. However, at present, most batteries are working well beyond 6 years, even though a real estimation is currently under evaluation. The corresponding maintenance cost is consistent with the design plans anyway. The SDE is situated on the top surface, properly protected from rain and dust by an aluminium dome. The SDE implements the station controller, event timing, an interface to communication system and a Slow Control system for measuring temperatures, voltages and currents. An ancillary Tank Power Control Board (TPCB) monitors the power system operation and it is directly connected to 


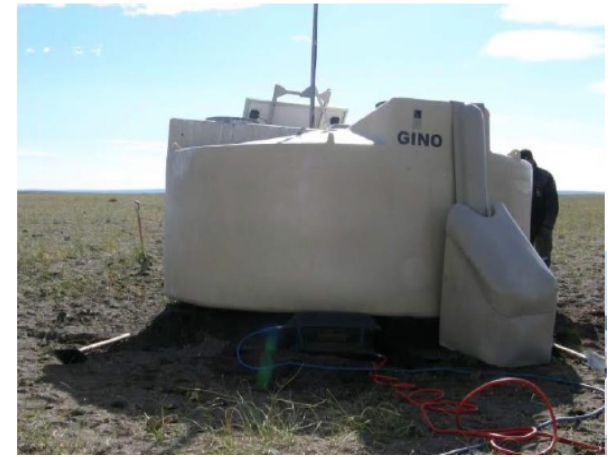

Figure 5: Damages caused by ground erosion and intervention in the field to repair an SD detector.

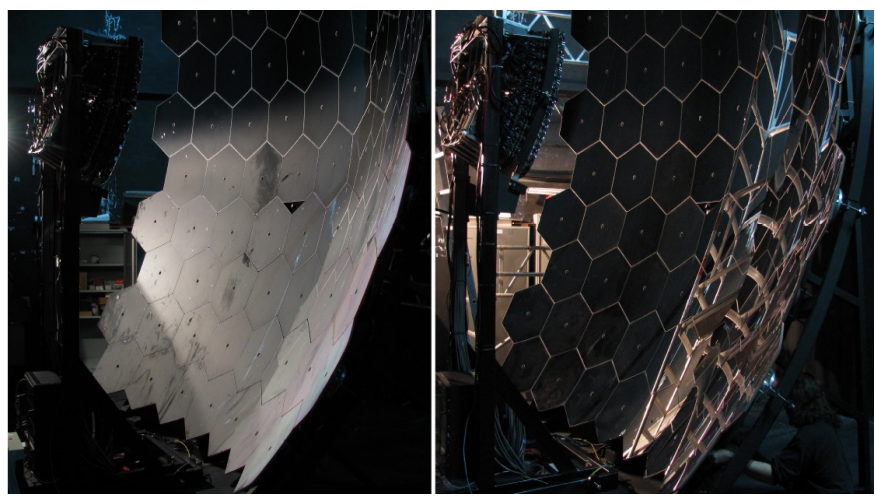

Figure 6: The whole mirror of one telescope at Coihueco site before (left) and after (right) a cleaning process (March 2011).

the SDE, able to control the power of the detectors; so that remotely, by way of the CDAS, the stations can be set in hibernation mode when the charge of batteries falls below a critical level, as occurred during the aforesaid occasion. The failure rate of electronic modules is about $1 \%$ per year; most of the electronics problems, mainly due to obsolescence, can be repaired and the components replaced on site by the local staff since all the spare parts are stored at the Observatory. The PMTs, operating at nominal gains between $2 \times 10^{5}$ and $3 \times 10^{6}$, are required to be linear within $5 \%$ up to $50 \mathrm{~mA}$ anode current. Their base, including the high voltage (HV) supply, is protected by silicone potting from the high humidity present in the tank. A LED flasher, mounted in a test port of the liner in a few stations, is used for testing remotely the PMT linearity. The failure rate is about $0.5 \%$ (20 PMTs per year) mainly due to problems of the HV modules and PMT bases as well as bad connections. Damages of the phototubes themselves (such as broken photo-catodes) require a total replacement whereas all other failures can be repaired on site. In the latest two years about a hundred of PMTs has been replaced by 9-in. diameter HZC Photonics XP1805 of Chinese manufacturing. The number of available spare PMTs, including the recent procurement of the chinese PMTs, is sufficient for about 10-15 more years of operation.

\section{Operations and maintenance of the detectors: the FD telescopes}

The atmospheric fluorescence telescopes are very sensitive to environmental factors and are not operated when the weather conditions become dangerous (wind speed more than $50 \mathrm{~km} / \mathrm{h}$, hail, snow) nor when the night sky brightness (mainly due to scattered moonlight) is too high. More generally the FD data-taking depends on different experimental conditions: weather situation, light levels, power failures or communications breakdowns, hardware malfunctions and software problems. Therefore, the telescopes have to be operated manually and the data-acquisition is organized in runs (Sec.4). However the use of a Slow Control System, being able to monitor detectors and weather conditions [7], can assure a secure remote operation; it is also capable of performing an orderly shutdown of the detectors in case of unexpected external events. Even though the telescopes are protected in their buildings, cleaning and maintenance has been required during years of operation. The FD maintenance is performed by the local staff of engineers and technicians with the regular help of expert technicians and physicists from other Auger institutions, specifically for the 
filter and mirror cleaning, power supplies maintenance and calibration. Concerning the telescopes themselves (diaphragm, corrector ring, camera body, PMTs), only very few failures have been detected over the operation time of the Observatory. The PMTs work at a nominal gain of $5 \times 10^{4}$ for standard operations. The electronics for each PMT is connected to a distribution board providing high (HV) and low (LV) voltages and receiving the output PMT signals. After a relatively fast loss of gain in the first two years of operation, the gain becomes more stable, thanks to more stringent procedures (since November 2006) to limit the FD exposure to moonlight. Starting that time, the observed loss of gain, averaged over all telescopes, is equal to $1.8 \%$ per year, close to the calculated mean yearly loss of $(1-1.5) \%$. The UV filter transmission $(\sim 80 \%$ between $330 \div 380 \mathrm{~nm})$ can be affected by the deposited dust layers. Therefore the cleaning of the UV filters from outside is being performed 3 times per year. The inner side of the filter, the corrector ring, and the curtain are cleaned less frequently, because they are not exposed to the outside environment. As regards the primary mirror, the average reflectivity of cleaned segments is above $90 \%$ (at $\lambda=370 \mathrm{~nm}$ ). Dry and wet methods have been adopted for cleaning the mirror tiles (Fig.6) with an improvement in their reflectivity: between $\leq 1 \%$ and $\sim 5 \%$, depending on the position of tiles (in the upper or bottom rows). Moreover the alignment of individual mirror tiles is cross-checked with a laser on site or additional hardware (external lasers) or software methods: only twice over the years a realignment of a telescope has been needed. The FDE requires a regular maintenance, in particular concerning the $\mathrm{HV}$ and LV power supplies, carried out by means of 2-3 trips per year performed primarily by expert technicians from other Auger institutions in collaboration with the local staff. In the recent years a total replacement of all the HV power systems that supply the telescopes has been fulfilled, due to their obsolescence after about 15 years of operation.

\section{Shifts of operations and data taking of the detectors: the FD shifts}

Regularly, the FD telescopes are controlled locally in a control room at the central campus of the Observatory in Malarguie by a shift-crew in charge of the preparation and monitoring of the FD run. The shift-crew is composed by 4-6 persons per FD shift in charge, scientists of the Pierre Auger Collaboration, assisted when necessary by the local personnel ${ }^{1}$. The crew changes run-by-run based upon a scheduled shift calendar within the Auger Collaboration. In general terms, a regular run consists in a set of steps from the first basic revisions, passing through electronic settings, making calibrations and handling the data taking, to the final closure activities. For each FD shift a detailed report (FD Shift Report) is written by the shift-crew. Typically the shift operations - included pre and post data taking activities - cover from 8 hours per night per 15-16 nights, during the austral summer (since October until February), requiring 4 shifters up to to 14 hours per night per 18 nights, during the austral winter (since March until September), requiring 6 shifters, per lunar cycle (about two weeks, before and after the new moon): a total of about 61 shifters per year is required. Over time, the FD shifts turned into a well-structured and tried-and-tested detailed procedure [7]. At the early stage of the Observatory (2001-2004), the shifts were executed directly inside the building of first telescopes. A few years later (since 2005) it became feasible to execute them remotely from the central campus of the Observatory. In recent years, regular FD shifts are also executed remotely by

\footnotetext{
${ }^{1}$ During the COVID pandemic travel restrictions, the local shift was performed just by the local staff.
} 


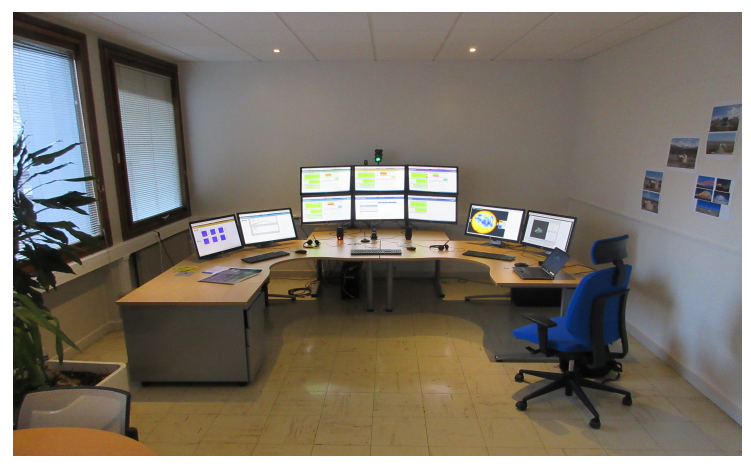

Figure 7: The remote control room installed in Grenoble (France) to execute FD shifts abroad.

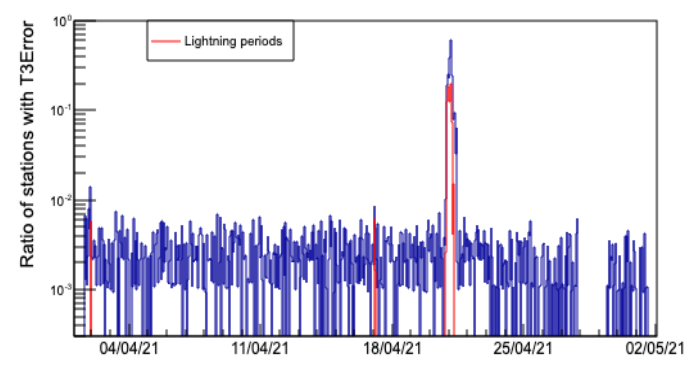

Figure 8: A typical diagram checked during a SD shift: the ratio of detectors showing a too high rate of $\mathrm{T} 3$ trigger errors, e.g. the rate of loss of $\mathrm{T} 3$ trigger events, correlated with lightning periods.

Auger teams in control rooms abroad, in sharing with the crew at the Observatory or managing the shift in exclusive charge from abroad: an operation mode primarily employed during the COVID19 pandemic due to lockdown restrictions. However the remote operation is only possible with dedicated local staff, being in standby for urgency calls from remote shifters. Due to casual network failures, for example, the local staff has to be ready for immediate response at the Observatory at any time during nights. The idea of the FD remote shifts was developed starting from 2011 when the first remote control room was installed at the Bergische Universität in Wuppertal, Germany and first tests were performed successfully. Over following years, 18 remote control rooms (Fig.7) all over the world have been installed offering the same functionality as the control room in Malargüe. The fulfillment of remote shift control rooms permitted a substantial reduction by up to $60 \%$ in the number of shifters needed locally at the Observatory in Malargüe, saving significant traveling time and costs. Moreover, during the COVID pandemic, the opportunity of remote shifts abroad allowed to have operation and data taking with the FD telescopes over the whole period, except for one shift that has been cancelled.

\section{Shifts of control and monitoring of the detectors: the SD shifts}

Before installing the SD shifts, the SD Array was checked and monitored regularly by a few SD experts. However, for a continuous monitoring of the SD detectors, starting from January 2019, regular SD shifts were established. They are very useful to check the short and long term performances of the SD Array. They represent a mean for each collaborator to keep contact with the Observatory, to understand low level data, the detector functioning, as for the FD telescopes. In addition, the SD shift represents a good opportunity, mainly for the younger students in Auger Collaboration, to look at SD events and to reconstruct a few of them at a basic level. The SD shifts have been gradually complemented over the following months and at present they are regularly and smoothly operated. Each SD shift can be carried out remotely at least one shifter by one Auger institution. The shifters change once every two weeks, based on a scheduled shift pattern within the Auger Collaboration. The SD shift requires a daily work, demanding less than one hour per day, during a 2 weeks period. It consists in a set of steps: from checking the status of the array (Black and Grey Tanks) to sanity checks on the acquired data, if they have been properly produced and 
are available. Pre-processed monthly files are used to assess the quality of the data taking, with clear indicators on trigger rates, bad periods ${ }^{2}$, event rates and final checks concerning the 5 highest energy reconstructed events of the preceding period (two weeks before the occurring shift). On request - in case of alerts - special checks are performed regarding the water-Cherenkov detectors showing a too high rate of T3 trigger errors, that is to say the rate of loss of T3 trigger events ${ }^{3}$, most likely due to strong lightning in the Array (Fig.8). Also for each SD shift a detailed report (SD Shift Report) is drawn up by the shifters.

\section{Concluding remarks}

The day-to-day operation of the Observatory demands 34 people on site, mostly highly qualified engineers and technicians and a not quantifiable number of them for maintenance campaigns over the years. Nightly FD shifts are covered by members of the Auger Collaboration, locally as well as in the different control rooms set up at many participating institutions, for a total of about 61 shifters per year. Recently, also day-by-day SD shifts are regularly operated remotely by Auger members abroad. Currently the SD Array and the FD telescopes are operating in a stable and efficient manner and shifts regularly carried out. The successful operation and regular maintenance of the Pierre Auger Observatory is possible thanks to the the strong dedication from the engineering, technical and administrative staff in Malarguie and also the personnel in charge from other Auger institutions, as well as all the FD and SD shifters.

\section{References}

[1] K. -H. Kampert, A. Watson Eur. Phys. J. H 37 (2012) 359-412.

[2] J. Abraham et al. Nucl. Instrum. Meth. A 523 (2004) 50-95.

[3] A. Aab et al. Nucl. Instrum. Meth. A 798 (2015) 172-213, [1502.01323v5].

[4] A. Aab et al. Science Reviews - from the end of the world (Argentina) 1 No.4 (2020) 8-33.

[5] I. Allekotte et al. Nucl. Instrum. Meth. A 586 (2008) 409-420.

[6] A. Abraham et al. Nucl. Instrum. Meth. A 620 (2010) 227-251.

[7] J. Rautenberg et al. [for the Pierre Auger coll.], Proc. 32nd Int. Cosmic Ray Conf., Beijing, China (2011), PoS(ICRC2011)5, [1107.4806v1].

[8] R. Sato et al. [for the Pierre Auger coll.], Proc. 32nd Int. Cosmic Ray Conf., Beijing, China (2011), PoS(ICRC2011)1, [1107.4806v1].

[9] K. Choi et al. [for the Pierre Auger coll.], Proc. 36thnd Int. Cosmic Ray Conf., Madison, Wisconsin, USA (2019), PoS(ICRC2019)174, [1909.09073].

\footnotetext{
${ }^{2}$ The bad periods are periods of time during which the event rate is not as expected. They are updated monthly and distinguished into two kinds: periods characterized by very high T3 error rates due to lightning, showing a deficit of events with respect to exposure, named as T3 bad periods, and the exclusion is done at the hours level and classic bad periods when the time interval between two events is not as expected and, in that case, the exclusion is done at the minute level.

${ }^{3}$ The SD rate of loss of T3 trigger events is defined as the ratio of stations in error divided by the total number of stations considered in the event.
} 


\section{The Pierre Auger Collaboration}

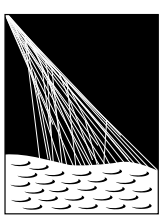

PIERRE

AUSGERVATORY

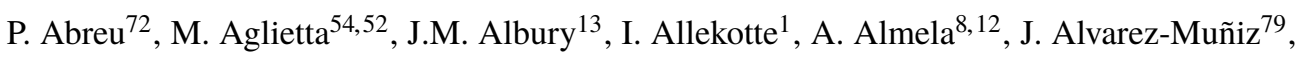
R. Alves Batista ${ }^{80}$, G.A. Anastasi ${ }^{63,52}$, L. Anchordoqui ${ }^{87}$, B. Andrada ${ }^{8}$, S. Andringa ${ }^{72}$, C. $\mathrm{Aramo}^{50}$, P.R. Araújo Ferreira ${ }^{42}$, J. C. Arteaga Velázquez ${ }^{67}$, H. Asorey ${ }^{8}$, P. Assis ${ }^{72}$, G. Avila ${ }^{11}$, A.M. Badescu ${ }^{75}$, A. Bakalova ${ }^{32}$, A. Balaceanu ${ }^{73}$, F. Barbato ${ }^{45,46}$, R.J. Barreira Luz $^{72}$, K.H. Becker ${ }^{38}$, J.A. Bellido ${ }^{13,69}$, C. Berat ${ }^{36}$, M.E. Bertaina ${ }^{63,52}$, X. Bertou ${ }^{1}$, P.L. Biermann ${ }^{b}$, V. Binet ${ }^{6}$, K. Bismark ${ }^{39,8}$, T. Bister ${ }^{42}$, J. Biteau ${ }^{37}$, J. Blazek ${ }^{32}$, C. Bleve ${ }^{36}$, M. Boháčová ${ }^{32}$, D. Boncioli ${ }^{57,46}$, C. Bonifazi ${ }^{9,26}$, L. Bonneau Arbeletche ${ }^{21}$, N. Borodai ${ }^{70}$, A.M. Botti ${ }^{8}$, J. Brack ${ }^{d}$, T. Bretz ${ }^{42}$, P.G. Brichetto Orchera ${ }^{8}$, F.L. Briechle ${ }^{42}$, P. Buchholz ${ }^{44}$, A. Bueno ${ }^{78}$, S. Buitink ${ }^{15}$, M. Buscemi ${ }^{47}$, M. Büsken ${ }^{39,8}$, K.S. Caballero-Mora ${ }^{66}$, L. Caccianiga ${ }^{59,49}$, F. Canfora ${ }^{80,81}$, I. Caracas ${ }^{38}$, J.M. Carceller ${ }^{78}$, R. Caruso ${ }^{58,47}$, A. Castellina ${ }^{54,52}$, F. Catalani ${ }^{19}$, G. Cataldi ${ }^{48}$, L. Cazon ${ }^{72}$, M. Cerda ${ }^{10}$, J.A. Chinellato ${ }^{22}$, J. Chudoba ${ }^{32}$, L. Chytka ${ }^{33}$, R.W. Clay ${ }^{13}$, A.C. Cobos Cerutti ${ }^{7}$, R. Colalillo ${ }^{60,50}$, A. Coleman ${ }^{93}$, M.R. Coluccia ${ }^{48}$, R. Conceição ${ }^{72}$, A. Condorelli ${ }^{45,46}$, G. Consolati ${ }^{49,55}$, F. Contreras ${ }^{11}$, F. Convenga ${ }^{56,48}$, D. Correia dos Santos $^{28}$, C.E. Covault ${ }^{85}$, S. Dasso ${ }^{5,3}$, K. Daumiller ${ }^{41}$, B.R. Dawson ${ }^{13}$, J.A. Day ${ }^{13}$, R.M. de Almeida ${ }^{28}$, J. de Jesús ${ }^{8,41}$, S.J. de Jong ${ }^{80,81}$, G. De Mauro ${ }^{80,81}$, J.R.T. de Mello Neto ${ }^{26,27}$, I. De Mitri ${ }^{45,46}$, J. de Oliveira ${ }^{18}$, D. de Oliveira Franco ${ }^{22}$, F. de Palma ${ }^{56,48}$, V. de Souza $^{20}$, E. De Vito ${ }^{56,48}$, M. del Río ${ }^{11}$, O. Deligny ${ }^{34}$, L. Deval ${ }^{41,8}$, A. di Matteo ${ }^{52}$, C. Dobrigkeit ${ }^{22}$, J.C. D'Olivo ${ }^{68}$, L.M. Domingues Mendes ${ }^{72}$, R.C. dos Anjos $^{25}$, D. dos $\operatorname{Santos}^{28}$, M.T. Dova ${ }^{4}$, J. Ebr ${ }^{32}$, R. Engel ${ }^{39,41}$, I. Epicoco ${ }^{56,48}$, M. Erdmann ${ }^{42}$, C.O. Escobar ${ }^{a}$, A. Etchegoyen ${ }^{8,12}$, H. Falcke ${ }^{80,82,81}$, J. Farmer ${ }^{92}$, G. Farrar ${ }^{90}$, A.C. Fauth ${ }^{22}$, N. Fazzini ${ }^{a}$, F. Feldbusch ${ }^{40}$, F. Fenu ${ }^{54,52}$, B. Fick ${ }^{89}$, J.M. Figueira ${ }^{8}$,

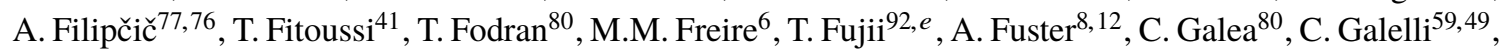
B. García ${ }^{7}$, A.L. Garcia Vegas ${ }^{42}$, H. Gemmeke ${ }^{40}$, F. Gesualdi ${ }^{8,41}$, A. Gherghel-Lascu ${ }^{73}$, P.L. Ghia ${ }^{34}$, U. Giaccari ${ }^{80}$, M. Giammarchi ${ }^{49}$, J. Glombitza ${ }^{42}$, F. Gobbi ${ }^{10}$, F. Gollan ${ }^{8}$, G. Golup ${ }^{1}$, M. Gómez Berisso ${ }^{1}$, P.F. Gómez Vitale ${ }^{11}$, J.P. Gongora ${ }^{11}$, J.M. González ${ }^{1}$, N. González ${ }^{14}$, I. Goos ${ }^{1,41}$, D. Góra ${ }^{70}$, A. Gorgi ${ }^{54,52}$,

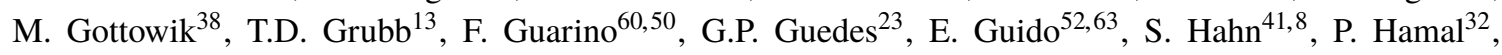
M.R. Hampel ${ }^{8}$, P. Hansen ${ }^{4}$, D. Harari ${ }^{1}$, V.M. Harvey ${ }^{13}$, A. Haungs ${ }^{41}$, T. Hebbeker ${ }^{42}$, D. Heck ${ }^{41}$, G.C. Hill ${ }^{13}$, C. Hojvat ${ }^{a}$, J.R. Hörandel ${ }^{80,81}$, P. Horvath ${ }^{33}$, M. Hrabovský ${ }^{33}$, T. Huege ${ }^{41,15}$, A. Insolia ${ }^{58,47}$, P.G. Isar ${ }^{74}$, P. Janecek ${ }^{32}$, J.A. Johnsen ${ }^{86}$, J. Jurysek ${ }^{32}$, A. Kääpä ${ }^{38}$, K.H. Kampert ${ }^{38}$, N. Karastathis ${ }^{41}$, B. Keilhauer ${ }^{41}$, J. Kemp ${ }^{42}$, A. Khakurdikar ${ }^{80}$, V.V. Kizakke Covilakam ${ }^{8,41}$, H.O. Klages ${ }^{41}$, M. Kleifges ${ }^{40}$, J. Kleinfeller ${ }^{10}$, M. Köpke ${ }^{39}$, N. Kunka ${ }^{40}$, B.L. Lago ${ }^{17}$, R.G. Lang ${ }^{20}$, N. Langner ${ }^{42}$, M.A. Leigui de Oliveira ${ }^{24}$, V. Lenok ${ }^{41}$, A. Letessier-Selvon ${ }^{35}$, I. Lhenry-Yvon ${ }^{34}$, D. Lo Presti ${ }^{58,47}$, L. Lopes ${ }^{72}$, R. López ${ }^{64}$, L. Lu ${ }^{94}$, Q. Luce ${ }^{39}$, J.P. Lundquist ${ }^{76}$, A. Machado Payeras ${ }^{22}$, G. Mancarella ${ }^{56,48}$, D. Mandat ${ }^{32}$, B.C. Manning ${ }^{13}$, J. Manshanden ${ }^{43}$, P. Mantsch ${ }^{a}$, S. Marafico ${ }^{34}$, A.G. Mariazzi ${ }^{4}$, I.C. Mariş ${ }^{14}$, G. Marsella ${ }^{61,47}$, D. Martello ${ }^{56,48}$, S. Martinelli ${ }^{41,8}$, O. Martínez Bravo ${ }^{64}$, M. Mastrodicasa ${ }^{57,46}$, H.J. Mathes ${ }^{41}$, J. Matthews ${ }^{88}$, G. Matthiae ${ }^{62,51}$, E. Mayotte ${ }^{38}$, P.O. Mazur ${ }^{a}$, G. Medina-Tanco ${ }^{68}$, D. Melo ${ }^{8}$, A. Menshikov ${ }^{40}$, K.-D. Merenda ${ }^{86}$, S. Michal ${ }^{33}$, M.I. Micheletti ${ }^{6}$, L. Miramonti ${ }^{59,49}$, S. Mollerach ${ }^{1}$, F. Montanet ${ }^{36}$, C. Morello ${ }^{54,52}$, M. Mostafá ${ }^{91}$, A.L. Müller ${ }^{8}$, M.A. Muller ${ }^{22}$, K. Mulrey ${ }^{15}$, R. Mussa ${ }^{52}$, M. Muzio ${ }^{90}$, W.M. Namasaka ${ }^{38}$, A. Nasr-Esfahani ${ }^{38}$, L. Nellen ${ }^{68}$, M. NiculescuOglinzanu $^{73}$, M. Niechciol ${ }^{44}$, D. Nitz ${ }^{89}$, D. Nosek ${ }^{31}$, V. Novotny ${ }^{31}$, L. Nožka ${ }^{33}$, A Nucita ${ }^{56,48}$, L.A. Núñez ${ }^{30}$, M. Palatka ${ }^{32}$, J. Pallotta ${ }^{2}$, P. Papenbreer ${ }^{38}$, G. Parente ${ }^{79}$, A. Parra ${ }^{64}$, J. Pawlowsky ${ }^{38}$, M. Pech ${ }^{32}$, F. Pedreira ${ }^{79}$, J. Pȩkala ${ }^{70}$, R. Pelayo ${ }^{65}$, J. Peña-Rodriguez ${ }^{30}$, E.E. Pereira Martins ${ }^{39,8}$, J. Perez Armand ${ }^{21}$, C. Pérez

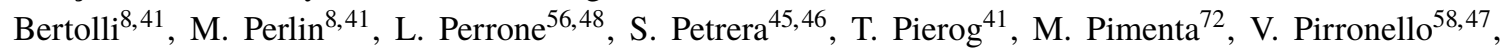
M. Platino ${ }^{8}$, B. Pont $^{80}$, M. Pothast ${ }^{81,80}$, P. Privitera ${ }^{92}$, M. Prouza $^{32}$, A. Puyleart ${ }^{89}$, S. Querchfeld ${ }^{38}$, J. Rautenberg ${ }^{38}$, D. Ravignani ${ }^{8}$, M. Reininghaus ${ }^{41,8}$, J. Ridky ${ }^{32}$, F. Riehn ${ }^{72}$, M. Risse ${ }^{44}$, V. Rizi ${ }^{5746}$, W. Rodrigues de Carvalho ${ }^{21}$, J. Rodriguez Rojo ${ }^{11}$, M.J. Roncoroni ${ }^{8}$, S. Rossoni ${ }^{43}$, M. Roth ${ }^{41}$, E. Roulet ${ }^{1}$, A.C. Rovero ${ }^{5}$, P. Ruehl ${ }^{44}$, A. Saftoiu ${ }^{73}$, F. Salamida ${ }^{57,46}$, H. Salazar ${ }^{64}$, G. Salina ${ }^{51}$, J.D. Sanabria Gomez ${ }^{30}$, F. Sánchez ${ }^{8}$, E.M. Santos ${ }^{21}$, E. Santos ${ }^{32}$, F. Sarazin ${ }^{86}$, R. Sarmento ${ }^{72}$, C. Sarmiento-Cano ${ }^{8}$, R. Sato ${ }^{11}$, P. Savina ${ }^{56,48,34,94}$, C.M. Schäfer ${ }^{41}$, V. Scherini ${ }^{56,48}$, H. Schieler ${ }^{41}$, M. Schimassek ${ }^{39,8}$, M. Schimp ${ }^{38}$, 
F. Schlüter ${ }^{41,8}$, D. Schmidt ${ }^{39}$, O. Scholten ${ }^{84,15}$, P. Schovánek ${ }^{32}$, F.G. Schröder ${ }^{93,41}$, S. Schröder ${ }^{38}$, J. Schulte ${ }^{42}$, S.J. Sciutto ${ }^{4}$, M. Scornavacche ${ }^{8,41}$, A. Segreto ${ }^{53,47}$, S. Sehgal ${ }^{38}$, R.C. Shellard ${ }^{16}$, G. Sigl ${ }^{43}$, G. Silli ${ }^{8,41}$, O. Sima ${ }^{73, f}$, R. Šmída ${ }^{92}$, P. Sommers ${ }^{91}$, J.F. Soriano ${ }^{87}$, J. Souchard ${ }^{36}$, R. Squartini ${ }^{10}$, M. Stadelmaier ${ }^{41,8}$, D. Stanca $^{73}$, S. Stanič ${ }^{76}$, J. Stasielak ${ }^{70}$, P. Stassi ${ }^{36}$, A. Streich ${ }^{39,8}$, M. Suárez-Durán ${ }^{14}$, T. Sudholz ${ }^{13}$, T. Suomijärvi ${ }^{37}$, A.D. Supanitsky ${ }^{8}$, Z. Szadkowski ${ }^{71}$, A. Tapia ${ }^{29}$, C. Taricco $^{63,52}$, C. Timmermans ${ }^{81,80}$, O. Tkachenko ${ }^{41}$, P. Tobiska ${ }^{32}$, C.J. Todero Peixoto ${ }^{19}$, B. Tomé ${ }^{72}$, Z. Torrès ${ }^{36}$, A. Travaini ${ }^{10}$, P. Travnicek $^{32}$, C. Trimarelli ${ }^{57,46}$, M. Tueros ${ }^{4}$, R. Ulrich ${ }^{41}$, M. Unger ${ }^{41}$, L. Vaclavek ${ }^{33}$, M. Vacula ${ }^{33}$, J.F. Valdés Galicia ${ }^{68}$, L. Valore ${ }^{60,50}$, E. Varela ${ }^{64}$, A. Vásquez-Ramírez ${ }^{30}$, D. Veberič ${ }^{41}$, C. Ventura ${ }^{27}$, I.D. Vergara Quispe ${ }^{4}$,

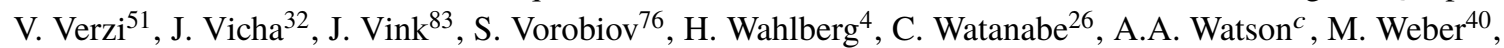
A. Weindl ${ }^{41}$, L. Wiencke ${ }^{86}$, H. Wilczyński ${ }^{70}$, M. Wirtz ${ }^{42}$, D. Wittkowski ${ }^{38}$, B. Wundheiler ${ }^{8}$, A. Yushkov $^{32}$, O. Zapparrata ${ }^{14}$, E. Zas ${ }^{79}$, D. Zavrtanik ${ }^{76,77}$, M. Zavrtanik ${ }^{77,76}$, L. Zehrer ${ }^{76}$

${ }^{1}$ Centro Atómico Bariloche and Instituto Balseiro (CNEA-UNCuyo-CONICET), San Carlos de Bariloche, Argentina

${ }^{2}$ Centro de Investigaciones en Láseres y Aplicaciones, CITEDEF and CONICET, Villa Martelli, Argentina

${ }^{3}$ Departamento de Física and Departamento de Ciencias de la Atmósfera y los Océanos, FCEyN, Universidad de Buenos Aires and CONICET, Buenos Aires, Argentina

${ }^{4}$ IFLP, Universidad Nacional de La Plata and CONICET, La Plata, Argentina

${ }^{5}$ Instituto de Astronomía y Física del Espacio (IAFE, CONICET-UBA), Buenos Aires, Argentina

${ }^{6}$ Instituto de Física de Rosario (IFIR) - CONICET/U.N.R. and Facultad de Ciencias Bioquímicas y Farmacéuticas U.N.R., Rosario, Argentina

${ }^{7}$ Instituto de Tecnologías en Detección y Astropartículas (CNEA, CONICET, UNSAM), and Universidad Tecnológica Nacional - Facultad Regional Mendoza (CONICET/CNEA), Mendoza, Argentina

${ }^{8}$ Instituto de Tecnologías en Detección y Astropartículas (CNEA, CONICET, UNSAM), Buenos Aires, Argentina

${ }^{9}$ International Center of Advanced Studies and Instituto de Ciencias Físicas, ECyT-UNSAM and CONICET, Campus Miguelete - San Martín, Buenos Aires, Argentina

${ }^{10}$ Observatorio Pierre Auger, Malargüe, Argentina

11 Observatorio Pierre Auger and Comisión Nacional de Energía Atómica, Malargüe, Argentina

12 Universidad Tecnológica Nacional - Facultad Regional Buenos Aires, Buenos Aires, Argentina

${ }^{13}$ University of Adelaide, Adelaide, S.A., Australia

${ }^{14}$ Université Libre de Bruxelles (ULB), Brussels, Belgium

${ }^{15}$ Vrije Universiteit Brussels, Brussels, Belgium

${ }^{16}$ Centro Brasileiro de Pesquisas Fisicas, Rio de Janeiro, RJ, Brazil

${ }^{17}$ Centro Federal de Educação Tecnológica Celso Suckow da Fonseca, Nova Friburgo, Brazil

${ }^{18}$ Instituto Federal de Educação, Ciência e Tecnologia do Rio de Janeiro (IFRJ), Brazil

${ }^{19}$ Universidade de São Paulo, Escola de Engenharia de Lorena, Lorena, SP, Brazil

${ }^{20}$ Universidade de São Paulo, Instituto de Física de São Carlos, São Carlos, SP, Brazil

${ }^{21}$ Universidade de São Paulo, Instituto de Física, São Paulo, SP, Brazil

22 Universidade Estadual de Campinas, IFGW, Campinas, SP, Brazil

${ }^{23}$ Universidade Estadual de Feira de Santana, Feira de Santana, Brazil

${ }^{24}$ Universidade Federal do ABC, Santo André, SP, Brazil

25 Universidade Federal do Paraná, Setor Palotina, Palotina, Brazil

${ }^{26}$ Universidade Federal do Rio de Janeiro, Instituto de Física, Rio de Janeiro, RJ, Brazil

${ }^{27}$ Universidade Federal do Rio de Janeiro (UFRJ), Observatório do Valongo, Rio de Janeiro, RJ, Brazil

28 Universidade Federal Fluminense, EEIMVR, Volta Redonda, RJ, Brazil

${ }^{29}$ Universidad de Medellín, Medellín, Colombia

${ }^{30}$ Universidad Industrial de Santander, Bucaramanga, Colombia

${ }^{31}$ Charles University, Faculty of Mathematics and Physics, Institute of Particle and Nuclear Physics, Prague, Czech Republic

32 Institute of Physics of the Czech Academy of Sciences, Prague, Czech Republic

${ }^{33}$ Palacky University, RCPTM, Olomouc, Czech Republic 
${ }^{34}$ CNRS/IN2P3, IJCLab, Université Paris-Saclay, Orsay, France

${ }^{35}$ Laboratoire de Physique Nucléaire et de Hautes Energies (LPNHE), Sorbonne Université, Université de Paris, CNRSIN2P3, Paris, France

${ }^{36}$ Univ. Grenoble Alpes, CNRS, Grenoble Institute of Engineering Univ. Grenoble Alpes, LPSC-IN2P3, 38000 Grenoble, France

${ }^{37}$ Université Paris-Saclay, CNRS/IN2P3, IJCLab, Orsay, France

38 Bergische Universität Wuppertal, Department of Physics, Wuppertal, Germany

${ }^{39}$ Karlsruhe Institute of Technology (KIT), Institute for Experimental Particle Physics, Karlsruhe, Germany

${ }^{40}$ Karlsruhe Institute of Technology (KIT), Institut für Prozessdatenverarbeitung und Elektronik, Karlsruhe, Germany

${ }^{41}$ Karlsruhe Institute of Technology (KIT), Institute for Astroparticle Physics, Karlsruhe, Germany

${ }^{42}$ RWTH Aachen University, III. Physikalisches Institut A, Aachen, Germany

${ }^{43}$ Universität Hamburg, II. Institut für Theoretische Physik, Hamburg, Germany

44 Universität Siegen, Department Physik - Experimentelle Teilchenphysik, Siegen, Germany

${ }^{45}$ Gran Sasso Science Institute, L’Aquila, Italy

${ }^{46}$ INFN Laboratori Nazionali del Gran Sasso, Assergi (L’Aquila), Italy

${ }^{47}$ INFN, Sezione di Catania, Catania, Italy

${ }^{48}$ INFN, Sezione di Lecce, Lecce, Italy

${ }^{49}$ INFN, Sezione di Milano, Milano, Italy

50 INFN, Sezione di Napoli, Napoli, Italy

51 INFN, Sezione di Roma "Tor Vergata”, Roma, Italy

52 INFN, Sezione di Torino, Torino, Italy

53 Istituto di Astrofisica Spaziale e Fisica Cosmica di Palermo (INAF), Palermo, Italy

54 Osservatorio Astrofisico di Torino (INAF), Torino, Italy

55 Politecnico di Milano, Dipartimento di Scienze e Tecnologie Aerospaziali , Milano, Italy

56 Università del Salento, Dipartimento di Matematica e Fisica “E. De Giorgi”, Lecce, Italy

${ }^{57}$ Università dell'Aquila, Dipartimento di Scienze Fisiche e Chimiche, L’Aquila, Italy

${ }^{58}$ Università di Catania, Dipartimento di Fisica e Astronomia, Catania, Italy

${ }^{59}$ Università di Milano, Dipartimento di Fisica, Milano, Italy

${ }^{60}$ Università di Napoli "Federico II", Dipartimento di Fisica "Ettore Pancini”, Napoli, Italy

${ }^{61}$ Università di Palermo, Dipartimento di Fisica e Chimica "E. Segrè”, Palermo, Italy

62 Università di Roma “Tor Vergata", Dipartimento di Fisica, Roma, Italy

${ }^{63}$ Università Torino, Dipartimento di Fisica, Torino, Italy

64 Benemérita Universidad Autónoma de Puebla, Puebla, México

${ }^{65}$ Unidad Profesional Interdisciplinaria en Ingeniería y Tecnologías Avanzadas del Instituto Politécnico Nacional (UPIITA-IPN), México, D.F., México

${ }^{66}$ Universidad Autónoma de Chiapas, Tuxtla Gutiérrez, Chiapas, México

${ }^{67}$ Universidad Michoacana de San Nicolás de Hidalgo, Morelia, Michoacán, México

${ }^{68}$ Universidad Nacional Autónoma de México, México, D.F., México

${ }^{69}$ Universidad Nacional de San Agustin de Arequipa, Facultad de Ciencias Naturales y Formales, Arequipa, Peru

${ }^{70}$ Institute of Nuclear Physics PAN, Krakow, Poland

${ }^{71}$ University of Łódź, Faculty of High-Energy Astrophysics,Lódź, Poland

${ }^{72}$ Laboratório de Instrumentação e Física Experimental de Partículas - LIP and Instituto Superior Técnico - IST, Universidade de Lisboa - UL, Lisboa, Portugal

73 "Horia Hulubei” National Institute for Physics and Nuclear Engineering, Bucharest-Magurele, Romania

${ }^{74}$ Institute of Space Science, Bucharest-Magurele, Romania

75 University Politehnica of Bucharest, Bucharest, Romania

${ }^{76}$ Center for Astrophysics and Cosmology (CAC), University of Nova Gorica, Nova Gorica, Slovenia

${ }^{77}$ Experimental Particle Physics Department, J. Stefan Institute, Ljubljana, Slovenia

${ }^{78}$ Universidad de Granada and C.A.F.P.E., Granada, Spain

${ }^{79}$ Instituto Galego de Física de Altas Enerxías (IGFAE), Universidade de Santiago de Compostela, Santiago de Compostela, Spain

${ }^{80}$ IMAPP, Radboud University Nijmegen, Nijmegen, The Netherlands 
${ }^{81}$ Nationaal Instituut voor Kernfysica en Hoge Energie Fysica (NIKHEF), Science Park, Amsterdam, The Netherlands

${ }^{82}$ Stichting Astronomisch Onderzoek in Nederland (ASTRON), Dwingeloo, The Netherlands

${ }^{83}$ Universiteit van Amsterdam, Faculty of Science, Amsterdam, The Netherlands

${ }^{84}$ University of Groningen, Kapteyn Astronomical Institute, Groningen, The Netherlands

${ }^{85}$ Case Western Reserve University, Cleveland, OH, USA

${ }^{86}$ Colorado School of Mines, Golden, CO, USA

${ }^{87}$ Department of Physics and Astronomy, Lehman College, City University of New York, Bronx, NY, USA

${ }^{88}$ Louisiana State University, Baton Rouge, LA, USA

${ }^{89}$ Michigan Technological University, Houghton, MI, USA

${ }^{90}$ New York University, New York, NY, USA

${ }^{91}$ Pennsylvania State University, University Park, PA, USA

92 University of Chicago, Enrico Fermi Institute, Chicago, IL, USA

93 University of Delaware, Department of Physics and Astronomy, Bartol Research Institute, Newark, DE, USA

94 University of Wisconsin-Madison, Department of Physics and WIPAC, Madison, WI, USA

${ }^{a}$ Fermi National Accelerator Laboratory, Fermilab, Batavia, IL, USA

${ }^{b}$ Max-Planck-Institut für Radioastronomie, Bonn, Germany

${ }^{c}$ School of Physics and Astronomy, University of Leeds, Leeds, United Kingdom

${ }^{d}$ Colorado State University, Fort Collins, CO, USA

${ }^{e}$ now at Hakubi Center for Advanced Research and Graduate School of Science, Kyoto University, Kyoto, Japan

${ }^{f}$ also at University of Bucharest, Physics Department, Bucharest, Romania 